01

\title{
Изотопные эффекты в спектрах комплексов с водородными связями. Расчет структуры и колебательных спектров поглощения комплексов $\mathrm{H}_{2} \mathrm{O}$. . .HF, $\mathrm{H}_{2} \mathrm{O}$. . .DF, $\mathrm{D}_{2} \mathrm{O}$. . .HF и $\mathrm{D}_{2} \mathrm{O}$. . .DF
}

\author{
(C) В.П. Булычев, М.В. Бутурлимова, К.Г. Тохадзе
}

Санкт-Петербургский государственный университет, 199034 Санкт-Петербург, Россия

e-mail: v.bulychev@spbu.ru

Поступила в редакцию 30.04.2021 г.

В окончательной редакции 12.06.2021 г.

Принята к публикации 20.06.2021 г.

Рассчитаны частоты и интенсивности ИК полос поглощения комплексов с водородными связями $\mathrm{H}_{2} \mathrm{O} \ldots \mathrm{HF}, \mathrm{H}_{2} \mathrm{O} \ldots \mathrm{DF}, \mathrm{D}_{2} \mathrm{O}$...HF и $\mathrm{D}_{2} \mathrm{O}$. . DF с использованием колебательной теории возмущений второго порядка. При определении равновесной конфигурации, поверхностей потенциальной энергии и дипольного момента этих комплексов, а также при вычислении спектральных параметров электронные волновые функции вычислялись по методу MP2/aug-cc-pVTZ с учетом ошибки наложения базисных наборов мономеров. Показано, что при образовании комплексов более всего меняются частоты и интенсивности валентного колебания молекул $\mathrm{HF}(\mathrm{DF})$ и интенсивности валентных колебаний молекул $\mathrm{H}_{2} \mathrm{O}\left(\mathrm{D}_{2} \mathrm{O}\right)$. Вариационный расчет либрации молекулы воды в потенциале с двумя минимумами объяснил причину более высокого значения частоты фундаментального перехода по моде $v_{1}(\mathrm{H}-\mathrm{F})$ по сравнению с частотой горячего перехода по этой моде с первого возбужденного состояния либрации. Выполнен анализ зависимости межмодового ангармонического взаимодействия от изотопного замещения.

Ключевые слова: водородная связь, расчеты спектров молекулярных комплексов, ангармонические взаимодействия, изотопные эффекты.

DOI: $10.21883 /$ OS.2021.10.51487.2255-21

\section{Введение}

Эта работа посвящена памяти профессора М.О. Буланина, многие плодотворные идеи которого использовались в наших исследованиях спектров атомно-молекулярных систем [1-3]. Комплекс $\mathrm{H}_{2} \mathrm{O}$...НF, спектр которого рассчитывается в данной статье, является простейшим комплексом, содержащим межмолекулярную водородную связь О. . .HF. Поэтому его можно рассматривать в качестве модельной системы при исследовании таких комплексов. Спектр комплекса $\mathrm{H}_{2} \mathrm{O}$. . .HF достаточно часто был объектом экспериментальных и теоретических исследований, но его изотопологи, содержащие ядра дейтерия, изучались намного реже.

Значительная информация о равновесной ядерной конфигурации комплекса $\mathrm{H}_{2} \mathrm{O}$...HF, его вращательных постоянных и низкочастотном спектре была получена методами вращательной спектроскопии [4,5]. Анализ вращательных спектров $[4,5]$ показал, что комплекс $\mathrm{H}_{2} \mathrm{O}$. . HF имеет симметрию точечной группы $C_{s}$ (рис. 1). На рис. 1 ось $z$ проходит через центры тяжести мономеров, а ось $y$ лежит в плоскости симметрии комплекса. Молекула воды может совершать либрацию по углу $\theta_{1}$ относительно плоской конфигурации, которая иногда называется инверсионным колебанием. В [5] для либрации молекулы воды в комплексе $\mathrm{H}_{2} \mathrm{O}$. ..НF была получена эмпирическая функция потенциальной энергии с двумя эквивалентными минимумами, повернутыми друг относительно друга на $92 \pm 16^{\circ}$ и разделенными инверсионным барьером высотой $126 \pm 70 \mathrm{~cm}^{-1}$. Легкая проницаемость барьера позволяет использовать группу симметрии $C_{2 v}$ для классификации колебательных состояний комплекса $\mathrm{H}_{2} \mathrm{O}$...HF. Из вращательных спектров в [5] были получены оценки частот фундаментальных переходов по низкочастотным колебаниям комплекса: $v_{3}\left(\mathrm{~A}_{1}\right)=176 \pm 15 \mathrm{~cm}^{-1}$ - валентное колебание водородной связи, $v_{6}\left(\mathrm{~B}_{1}\right)=157 \pm 10 \mathrm{~cm}^{-1}$ - либрация $\mathrm{H}_{2} \mathrm{O}$ в плоскости $x z, v_{6}\left(\mathrm{~B}_{2}\right)=64 \pm 10 \mathrm{~cm}^{-1}$ — либрация $\mathrm{H}_{2} \mathrm{O}$ в плоскости $y z$ и частота первого обертона $v_{6}\left(\mathrm{~B}_{2}\right)=267 \pm 35 \mathrm{~cm}^{-1}$.

В работах [6,7] были зарегистрированы некоторые колебательные полосы поглощения комплекса

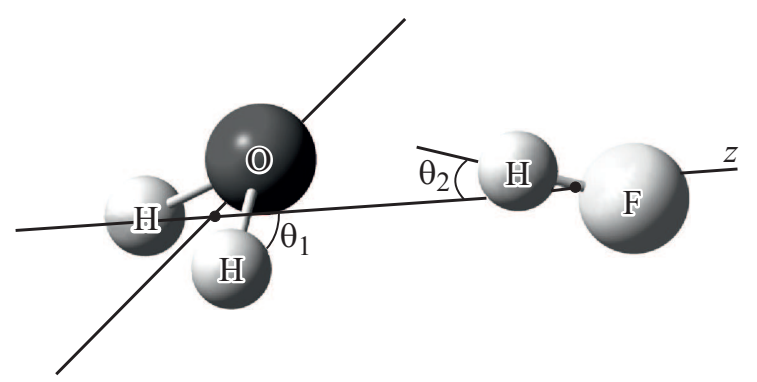

Рис. 1. Равновесная геометрия комплекса $\mathrm{H}_{2} \mathrm{O}$. . НF. Ось $z$ проходит через центры масс фрагментов $\mathrm{H}_{2} \mathrm{O}$ и $\mathrm{HF}$. 
$\mathrm{H}_{2} \mathrm{O}$...HF в газовой фазе и сделана попытка их интерпретации. Были получены следующие оценки частот (в $\left.\mathrm{cm}^{-1}\right): v_{1}\left(\mathrm{~A}_{1}\right)=3608 \pm 2$ - валентное колебание $\mathrm{H}-\mathrm{F}, \quad v_{6}\left(\mathrm{~B}_{1}\right)=170 \pm 50, \quad v_{6}\left(\mathrm{~B}_{2}\right)=145 \pm 50$, $v_{3}\left(\mathrm{~A}_{1}\right)=180 \pm 30$ - валентное колебание водородной связи, $v_{5}\left(\mathrm{~B}_{1}\right)=696 \pm 30$ - либрация НF в плоскости $x z, v_{5}\left(\mathrm{~B}_{2}\right)=666 \pm 30$ - либрация HF в плоскости $y z$. Для глубины минимума потенциальной кривой водородной связи была получена оценка $30 \mathrm{~kJ} \cdot \mathrm{mol}^{-1}$. В [8] спектры поглощения комплекса $\mathrm{H}_{2} \mathrm{O}$...HF и его дейтерированных изотопологов изучались в аргоновой матрице при температуре $12 \mathrm{~K}$. Для частоты валентного колебания фтористого водорода в гетеродимерах были получены значения 3554.7 ( $\left.\mathrm{H}_{2} \mathrm{O} \ldots \mathrm{HF}\right)$, 3549.0 ( $\left.\mathrm{D}_{2} \mathrm{O} \ldots \mathrm{HF}\right), 2621.1\left(\mathrm{H}_{2} \mathrm{O} \ldots \mathrm{DF}\right)$ и $2613.4 \mathrm{~cm}^{-1}$ $\left(\mathrm{D}_{2} \mathrm{O}\right.$...DF). Были также зарегистрированы мультиплеты, связанные с либрационными колебаниями мономеров HF и DF в двух плоскостях.

Полоса поглощения комплекса $\mathrm{H}_{2} \mathrm{O}$. . .HF в области фундаментального перехода $v_{1}\left(\mathrm{~A}_{1}\right)$ в газовой фазе при комнатной температуре была получена в $[9,10]$. Спектры газовых смесей $\mathrm{H}_{2} \mathrm{O} / \mathrm{HF}$ были зарегистрированы при температуре $293 \mathrm{~K}$ в области $4000-3400 \mathrm{~cm}^{-1}$ с разрешением $0.2-0.02 \mathrm{~cm}^{-1}$ при помощи вакуумных фурьеспектрометров Bruker IFS-113v и Bruker IFS-120 HR. Спектры комплекса $\mathrm{H}_{2} \mathrm{O}$...HF в области валентной полосы поглощения $\mathrm{H}-\mathrm{F}$ были получены вычитанием рассчитанных спектров свободных молекул $\mathrm{H}_{2} \mathrm{O}$ и $\mathrm{HF}$ из экспериментальных спектров. Равновесная геометрия и поверхности потенциальной энергии и дипольного момента комплекса $\mathrm{H}_{2} \mathrm{O}$...НF были рассчитаны в приближении MP2 с набором атомных функций $\mathrm{TZV}++(2 \mathrm{df}, 2 \mathrm{p})$ без учета [9] и с учетом [10] ошибки наложения базисных наборов мономеров. Из вариационных решений одно-, двух- и трехмерных ангармонических колебательных задач в пространстве координат валентных колебаний мономера HF и водородной связи, а также либрационных движений молекул $\mathrm{H}_{2} \mathrm{O}$ и HF были определены параметры, необходимые для расчета полосы поглощения в области колебания $v_{1}\left(\mathrm{~A}_{1}\right)$. Экспериментально наблюдаемая полоса была реконструирована как суперпозиция колебательно-вращательных полос фундаментального перехода и горячих переходов с возбужденных состояний низкочастотных межмолекулярных колебаний. Теоретическая полоса поглощения комплекса, расположенная в области $3600-3720 \mathrm{~cm}^{-1}$, хорошо воспроизводила основные детали экспериментального спектра и объясняла их происхождение. Было показано, в частности, что фундаментальный переход $v_{1}=1 \leftarrow 0$ из основного колебательного состояния связан не с низкочастотным, а с центральным наиболее интенсивным максимумом. Это привело к новому значению частоты $3633.8 \mathrm{~cm}^{-1}$ для перехода $v_{1}=1 \leftarrow 0$ в комплексе $\mathrm{H}_{2} \mathrm{O}$. . .HF, которое больше ранее принятого значения $3608 \mathrm{~cm}^{-1}$ [7]. Положение фундаментального перехода в широкой полосе поглощения комплекса, уточненное впервые в [9], было позднее подтверждено в низкотемпературном эксперименте с молекулярными пучками [11]. В [10] структура и спектр поглощения комплекса $\mathrm{H}_{2} \mathrm{O}$...DF были рассчитаны теми же методами, что и характеристики комплекса $\mathrm{H}_{2} \mathrm{O}$...НF. Для $\mathrm{H}_{2} \mathrm{O}$...DF было предсказано значение $2689 \mathrm{~cm}^{-1}$ для частоты $v_{1}(\mathrm{DF})$ и менее структурированный вид полосы поглощения $v_{1}(\mathrm{DF})$ при комнатной температуре.

В теоретических работах, выполненных до 2000 г., структура комплекса $\mathrm{H}_{2} \mathrm{O}$...HF рассчитывалась, как правило, с наборами атомных функций умеренной полноты, а частоты колебаний определялись в гармоническом или одномерном ангармоническом приближениях [12-14]. Более поздние теоретические работы [15-17] были выполнены на более высоком ab initio уровне, но полный колебательный спектр этого комплекса не изучался. В [15] использовался метод $\operatorname{CCSD}(\mathrm{T})$ и очень полные наборы атомных функций. Было изучено влияние полноты наборов функций и ошибки наложения базисных наборов мономеров на равновесные геометрические параметры комплекса и энергию димеризации $\Delta E$. В наиболее точном варианте расчета были получены значения $r(\mathrm{OH})=0.9594 \AA, r(\mathrm{HF})=0.9357 \AA, r(\mathrm{~F} \ldots \mathrm{O})=2.6497 \AA$, $r(\mathrm{O} \ldots \mathrm{H})=1.7144 \AA$ и $\Delta E=-37.74 \mathrm{~kJ} \cdot \mathrm{mol}^{-1}$. В расчетах [16] использовался метод MP2 с набором атомных функций aug-cc-pV5Z и метод $\operatorname{CCSD}(\mathrm{T})$ с набором атомных функций aug-cc-pVTZ. Основной целью работы было максимально точное определение энергии диссоциации гетеродимера. Частоты колебаний рассчитывались как в гармоническом приближении, так и с учетом ангармонических поправок по теории возмущений второго порядка. Для энергии диссоциации $D_{e}$ гетеродимера было получено значение $37.53 \mathrm{~kJ} \cdot \mathrm{mol}^{-1}$. В [17] с использованием метода $\operatorname{CCSD}(\mathrm{T})$ и набора атомных функций aug-cc-pVTZ была рассчитана пятимерная поверхность потенциальной энергии для низкочастотных колебаний $\mathrm{H}_{2} \mathrm{O}$...HF при замороженных внутренних колебаниях мономеров. Вариационным методом были рассчитаны энергии и волновые функции низкочастотных межмолекулярных колебаний. Для энергии диссоциации $D_{e}$ комплекса было получено значение $36.61 \mathrm{~kJ} \cdot \mathrm{mol}^{-1}$.

В настоящей работе выполняются ab initio pacчеты комплексов $\mathrm{H}_{2} \mathrm{O}$...HF, $\mathrm{H}_{2} \mathrm{O}$...DF, $\mathrm{D}_{2} \mathrm{O}$...HF и $\mathrm{D}_{2} \mathrm{O}$. . . DF с использованием метода MP2/aug-cc-pVTZ с учетом ошибки наложения наборов атомных функций мономеров. Расчет спектральных параметров четырех указанных комплексов весьма желателен ввиду ограниченности экспериментальной информации о спектрах комплекса $\mathrm{H}_{2} \mathrm{O}$...НF и его изотопологов как в низкотемпературных матрицах, так и в газовой фазе, и отсутствия теоретических данных о спектрах поглощения комплексов $\mathrm{H}_{2} \mathrm{O}$...DF, $\mathrm{D}_{2} \mathrm{O}$...HF и $\mathrm{D}_{2} \mathrm{O}$...DF. Частоты и интенсивности всех фундаментальных и некоторых обертонных и составных переходов комплексов рассчитываются по методу колебательной теории возмущений второго порядка VPT2 $[18,19]$. Данные для моды $v_{6}\left(\mathrm{~B}_{2}\right)$ уточняются при помощи вариационного расчета. Объясняется причина аномального соот- 
ношения между частотами фундаментального $\left(v_{1}=1\right.$, $\left.v_{6}\left(\mathrm{~B}_{2}\right)=0\right) \leftarrow\left(v_{1}=0, v_{6}\left(\mathrm{~B}_{2}\right)=0\right)$ перехода и горячего $\left(v_{1}=1, v_{6}\left(\mathrm{~B}_{2}\right)=1\right) \leftarrow\left(v_{1}=0, v_{6}\left(\mathrm{~B}_{2}\right)=1\right)$ перехода в рассматриваемых комплексах.

\section{Метод расчета электронной структуры комплексов $\mathrm{H}_{2} \mathrm{O}$. . .HF, $\mathrm{H}_{2} \mathrm{O}$. . .DF, $D_{2} O$... HF и $D_{2} O$. . .DF}

В настоящей работе квантово-химические расчеты равновесной геометрии и электронной энергии комплексов $\mathrm{H}_{2} \mathrm{O}$. . HF, $\mathrm{H}_{2} \mathrm{O}$. . DF, $\mathrm{D}_{2} \mathrm{O}$. . . HF и $\mathrm{D}_{2} \mathrm{O}$. . . DF были выполнены по методу MP2/aug-cc-pVTZ с поправкой на ошибку наложения базисных наборов мономеров при помощи пакета программ Gaussian 16 [20]. Этот метод более точен, чем метод, использованный в $[9,10]$ для расчета комплекса $\mathrm{H}_{2} \mathrm{O}$....НF. Значения дипольных моментов и интенсивностей переходов между колебательными состояниями вычислялись с электронными волновыми функциями самосогласованного поля. Равновесная ядерная конфигурация комплексов обладает симметрией $C_{s}$ (рис. 1) и характеризуется параметрами $r(\mathrm{OH})=0.9623 \AA, r(\mathrm{HF})=0.9390 \AA, r(\mathrm{~F} \ldots \mathrm{O})=2.6617 \AA$, $r(\mathrm{O} \ldots \mathrm{H})=1.7232 \AA$ и $\Delta E=-34.96 \mathrm{~kJ} \cdot \mathrm{mol}^{-1}$; вклад учета ошибки наложения базисных наборов мономеров в $\Delta E$ составляет $2.48 \mathrm{~kJ} \cdot \mathrm{mol}^{-1}$. Эти значения находятся в хорошем согласии с наиболее точными литературными данными [15-17]. Дипольный момент комплекса в равновесной конфигурации равен $1.9685 \mathrm{D}$. Указанные варианты квантово-химических методов ab initio расчетов с учетом ошибки наложения базисных наборов мономеров были использованы также при выполнении гармонических, ангармонических и вариационных расчетов спектральных параметров.

\section{Результаты расчетов спектральных параметров и их обсуждение}

\section{Гармонические расчеты частот и интенсивностей спектральных переходов}

Частоты и интенсивности фундаментальных переходов в комплексах $\mathrm{H}_{2} \mathrm{O} \ldots \mathrm{HF}, \mathrm{H}_{2} \mathrm{O} \ldots \mathrm{DF}, \mathrm{D}_{2} \mathrm{O} \ldots \mathrm{HF}$ и $\mathrm{D}_{2} \mathrm{O} \ldots \mathrm{DF}$ были рассчитаны по колебательной теории возмущений второго порядка VPT2 [18,19] при помощи пакета программ Gaussian 16 [20]. Полученные результаты представлены в табл. 1. Для обозначения нормальных колебаний комплексов использованы неприводимые представления группы симметрии $C_{2 v}$ : $v_{1}\left(\mathrm{~A}_{1}\right)$ - валентное колебание $\mathrm{H}-\mathrm{F}(\mathrm{D}-\mathrm{F}), v_{2}\left(\mathrm{~A}_{1}\right)-$ деформационное колебание угла $\mathrm{HOH}$ (DOD) молекулы воды, $v_{3}\left(\mathrm{~A}_{1}\right)$ - валентное колебание водородной связи, $v_{4}\left(\mathrm{~A}_{1}\right)$ - симметричное валентное колебание длин связей $\mathrm{O}-\mathrm{H}(\mathrm{O}-\mathrm{D}), v_{4}\left(\mathrm{~B}_{2}\right)$ - антисимметричное валентное колебание длин связей $\mathrm{O}-\mathrm{H}(\mathrm{O}-\mathrm{D})$,
Таблица 1. Гармонические значения частот $\left(\mathrm{cm}^{-1}\right)$ и интенсивностей $\left(\mathrm{km} \cdot \mathrm{mol}^{-1}\right)$ (в скобках) фундаментальных ИК полос поглощения комплекса $\mathrm{H}_{2} \mathrm{O}$. . HF и его изотопологов

\begin{tabular}{c|l|l|l|l}
\hline Переход & $\mathrm{H}_{2} \mathrm{O} \ldots \mathrm{HF}$ & $\mathrm{H}_{2} \mathrm{O} \ldots \mathrm{DF}$ & $\mathrm{D}_{2} \mathrm{O} \ldots \mathrm{HF}$ & $\mathrm{D}_{2} \mathrm{O} \ldots \mathrm{DF}$ \\
\hline$v_{6}\left(\mathrm{~B}_{2}\right)$ & $219(34)$ & $216(35)$ & $176(62)$ & $174(59)$ \\
$\nu_{6}\left(\mathrm{~B}_{1}\right)$ & $242(0.1)$ & $227(0.03)$ & $181(0.02)$ & $175(0.1)$ \\
$v_{3}\left(\mathrm{~A}_{1}\right)$ & $261(162)$ & $255(143)$ & $241(52)$ & $238(49)$ \\
$v_{5}\left(\mathrm{~B}_{2}\right)$ & $701(156)$ & $516(121)$ & $695(134)$ & $506(86)$ \\
$v_{5}\left(\mathrm{~B}_{1}\right)$ & $823(123)$ & $629(76)$ & $798(111)$ & $592(64)$ \\
$v_{2}\left(\mathrm{~A}_{1}\right)$ & $1631(73)$ & $1631(75)$ & $1195(39)$ & $1195(41)$ \\
$v_{1}\left(\mathrm{~A}_{1}\right)$ & $3741(918)$ & $2717(472)$ & $3744(900)$ & $2714(467)$ \\
$v_{4}\left(\mathrm{~A}_{1}\right)$ & $3815(12)$ & $3813(22)$ & $2748(18)$ & $2751(15)$ \\
$v_{4}\left(\mathrm{~B}_{1}\right)$ & $3933(115)$ & $3933(115)$ & $2882(65)$ & $2882(65)$
\end{tabular}

$v_{5}\left(\mathrm{~B}_{1}\right)$ - либрация молекулы HF (DF) перпендикулярно плоскости симметрии комплекса $C_{s}, v_{5}\left(\mathrm{~B}_{2}\right)-$ либрация молекулы HF (DF) в плоскости симметрии комплекса $C_{s}, v_{6}\left(\mathrm{~B}_{1}\right)$ - либрация молекулы $\mathrm{H}_{2} \mathrm{O}\left(\mathrm{D}_{2} \mathrm{O}\right)$ перпендикулярно плоскости симметрии комплекса $C_{s}$, $v_{6}\left(\mathrm{~B}_{2}\right)$ - либрация молекулы $\mathrm{H}_{2} \mathrm{O}\left(\mathrm{D}_{2} \mathrm{O}\right)$ в плоскости симметрии комплекса $C_{s}$. Значения частот, полученных нами для комплекса $\mathrm{H}_{2} \mathrm{O}$...HF, находятся в хорошем согласии с данными расчета [16] по методу MP2 с более полным набором атомных функций aug-cc-pV5Z. Различие между результатами двух расчетов не превышает $5 \mathrm{~cm}^{-1}$ для большинства колебаний и составляет $11 \mathrm{~cm}^{-1}$ для $v_{5}\left(\mathrm{~B}_{2}\right), 14 \mathrm{~cm}^{-1}$ для $\nu_{5}\left(\mathrm{~B}_{1}\right)$ и $20 \mathrm{~cm}^{-1}$ для $v_{4}\left(\mathrm{~A}_{1}\right)$ и $v_{4}\left(\mathrm{~B}_{1}\right)$. Следует подчеркнуть, что нормальная координата деформационного колебания $v_{6}\left(\mathrm{~B}_{2}\right)$ описывает колебание только в одном из минимумов потенциальной энергии. Но барьер между двумя минимумами легко проницаем и молекула воды даже в основном колебательном состоянии должна иметь одинаковые вероятности нахождения в каждом из минимумов. Расчет, в котором деформационное колебание осуществляется только в одном из минимумов, искажает уровни энергии этого колебания и увеличивает взаимодействие этого колебания с валентным колебанием водородной связи. Анализ форм нормальных координат колебаний $\mathrm{H}_{2} \mathrm{O}$...HF с частотами 219 и $261 \mathrm{~cm}^{-1}$ и аналогичных колебаний других изотопологов показал, что каждое из этих колебаний является смесью валентного и деформационного колебаний, причем деформационное колебание происходит только в одном из минимумов. Поэтому гармонические значения частот и интенсивностей фундаментальных переходов по этим двум модам следует признать ненадежными. Что касается мономеров, то гар-

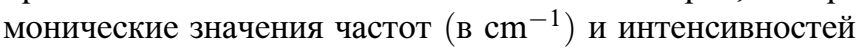
$\left(\right.$ в $\left.\mathrm{km} \cdot \mathrm{mol}^{-1}\right)$ (указаны в скобках), рассчитанные для них в приближении MP2/aug-cc-pVTZ, равны 4123 (121) для $\mathrm{HF}$ и 2989 (85) для $\mathrm{DF}, 1628$ (72), 3823 (6) и 3948 (75) для $\mathrm{H}_{2} \mathrm{O}$ и 1192 (38), 2756 (4) и 2893 (45) для $\mathrm{D}_{2} \mathrm{O}$. Таким образом, при образовании комплексов наибольшие изменения испытывают частоты и интен- 
Таблица 2. Ангармонические значения частот $\left(\mathrm{cm}^{-1}\right)$ и интенсивностей $\left(\mathrm{km} \cdot \mathrm{mol}^{-1}\right)$ (в скобках) ИК полос поглощения комплекса $\mathrm{H}_{2} \mathrm{O}$. . .HF и его изотопологов

\begin{tabular}{c|l|l|l|l}
\hline Переход & $\mathrm{H}_{2} \mathrm{O} \ldots \mathrm{HF}$ & $\mathrm{H}_{2} \mathrm{O} \ldots \mathrm{DF}$ & $\mathrm{D}_{2} \mathrm{O} \ldots \mathrm{HF}$ & $\mathrm{D}_{2} \mathrm{O} \ldots \mathrm{DF}$ \\
\hline$v_{6}\left(\mathrm{~B}_{2}\right)$ & $74(98)$ & $83(106)$ & $98(80)$ & $102(80)$ \\
$v_{6}\left(\mathrm{~B}_{1}\right)$ & $210(2)$ & $202(1)$ & $162(0.4)$ & $159(0.2)$ \\
$v_{3}\left(\mathrm{~A}_{1}\right)$ & $280(23)$ & $278(22)$ & $232(6)$ & $232(6)$ \\
$v_{5}\left(\mathrm{~B}_{2}\right)$ & $600(120)$ & $453(75)$ & $601(122)$ & $450(69)$ \\
$v_{5}\left(\mathrm{~B}_{1}\right)$ & $713(126)$ & $557(81)$ & $699(112)$ & $532(67)$ \\
$v_{2}\left(\mathrm{~A}_{1}\right)$ & $1588(70)$ & $1587(66)$ & $1172(32)$ & $1173(39)$ \\
$v_{1}\left(\mathrm{~A}_{1}\right)$ & $3598(666)$ & $2653(387)$ & $3596(702)$ & $2634(345)$ \\
$v_{4}\left(\mathrm{~A}_{1}\right)$ & $3655(47)$ & $3648(17)$ & $2663(15)$ & $2675(48)$ \\
$v_{4}\left(\mathrm{~B}_{1}\right)$ & $3756(99)$ & $3756(100)$ & $2786(58)$ & $2786(55)$ \\
$2 v_{5}\left(\mathrm{~B}_{2}\right)$ & $1109(32)$ & $815(14)$ & $1120(34)$ & $852(13)$ \\
$2 v_{5}\left(\mathrm{~B}_{1}\right)$ & $1319(26)$ & $1054(9)$ & $1298(35)$ & $1010(9)$
\end{tabular}

сивности валентного колебания молекул фтористого водорода и интенсивности валентных колебаний молекул воды.

\section{Ангармонические расчеты частот и интенсивностей спектральных переходов}

Результаты расчета частот и интенсивностей фундаментальных спектральных переходов в изучаемых комплексах по теории возмущений второго порядка VPT2 представлены в табл. 2. Там же указаны обертонные переходы, обладающие достаточно высокой интенсивностью. Ранее отмечалось [21,22], что применение теории возмущений второго порядка VPT2 может привести к ошибкам при рассмотрении низкочастотных колебаний, в частности, колебаний, имеющих несколько минимумов потенциальной энергии, разделенных низкими барьерами. Теория возмущений VPT2 использует колебательные нормальные координаты, полученные в гармоническом приближении. В предыдущем разделе было отмечено, что в случае комплекса $\mathrm{H}_{2} \mathrm{O} \ldots \mathrm{HF}$ и его изотопологов гармонический расчет описывает деформационное колебание $v_{6}\left(\mathrm{~B}_{2}\right)$ только в одном из двух минимумов потенциальной кривой. Это приводит к искаженному описанию волновых функций и уровней энергии моды $v_{6}\left(\mathrm{~B}_{2}\right)$ и валентного колебания водородной связи. Эти недостатки частично сохраняются и в ангармоническом расчете. Поэтому ангармонические значения частот и интенсивностей низкочастотных полос поглощения рассматриваемых комплексов, вычисленные по теории возмущений VPT2 $[18,19]$, следует также признать ненадежными. Остальные колебания комплексов $\mathrm{H}_{2} \mathrm{O}$...HF, $\mathrm{H}_{2} \mathrm{O}$...DF, $\mathrm{D}_{2} \mathrm{O} \ldots \mathrm{HF}$ и $\mathrm{D}_{2} \mathrm{O}$. . DF очень слабо взаимодействуют с колебанием $v_{6}\left(\mathrm{~B}_{2}\right)$, поэтому их спектральные параметры, рассчитанные по теории возмущений VPT2, более надежны. В случае комплекса $\mathrm{H}_{2} \mathrm{O}$...НF результаты табл. 2 довольно хорошо согласуются с данными, полученными в [16] при помощи комбинации результатов гармонического $\operatorname{CCSD}(\mathrm{T}) / \mathrm{aug}-\mathrm{cc}-\mathrm{pV} 5 \mathrm{Z}$ и ангармонического MP2/aug-cc-pV5Z расчетов. Значения частоты $v_{1}\left(\mathrm{~A}_{1}\right)$, приведенные в табл. 2 для четырех изотопологов, на $21-47 \mathrm{~cm}^{-1}$ выше результатов эксперимента в аргоновой матрице при $12 \mathrm{~K}$ [8]. Приблизительно такое же соотношение имеет место между нашими значениями частот либрационных колебаний $v_{5}\left(\mathrm{~B}_{1}\right)$ и $v_{5}\left(\mathrm{~B}_{2}\right)$ и соответствующими данными [8]. Расхождение между расчетными и экспериментальными результатами вполне объяснимо влиянием матричного окружения на колебания комплексов. При учете ангармоничности наибольшее уменьшение (на 65-170 $\mathrm{cm}^{-1}$ ) испытывают частоты валентных колебаний $v_{1}\left(\mathrm{~A}_{1}\right), v_{4}\left(\mathrm{~A}_{1}\right)$ и $v_{4}\left(\mathrm{~B}_{1}\right)$ во всех четырех комплексах, несколько менее (на 65-110 $\mathrm{cm}^{-1}$ ) понижаются частоты переходов $v_{5}\left(\mathrm{~B}_{2}\right)$ и $v_{5}\left(\mathrm{~B}_{1}\right)$, интенсивность переходов $v_{1}\left(\mathrm{~A}_{1}\right)$ понижается на $22-37 \%$ и значительно (до $30 \%$ ) падает интенсивность перехода $v_{5}\left(\mathrm{~B}_{2}\right)$. Интересно, что если в гармоническом приближении интенсивность перехода по симметричному валентному колебанию $v_{4}\left(\mathrm{~A}_{1}\right)$ связей $\mathrm{OH}$ (OD) молекулы воды больше в тех комплексах, в которых молекулы воды и фтористого водорода содержат разные изотопы водорода, то в ангармонических расчетах закономерность противоположная. В то же время частота и интенсивность перехода $v_{4}\left(\mathrm{~B}_{1}\right)$ практически не зависят от изотопного состава молекулы фтористого водорода при расчете как в гармоническом, так и ангармоническом приближениях. Ангармонические расчеты предсказывают возможность обнаружения обертонных полос поглощения, связанных с двумя либрационными колебаниями $v_{5}$, в области, доступной для инфракрасных спектроскопических исследований.

Дополнительную информацию о внутримодовом и межмодовом ангармонизме в комплексах дает анализ констант ангармонического взаимодействия $\chi_{i, j}$, определяемых согласно [19]. Здесь мы не будем анализировать ангармонические константы, имеющие отношение к трем низкочастотным модам, которые рассчитываются менее надежно. Из диагональных констант наибольшие значения имеют $\chi_{1,1}$, т.е. константы валентных колебаний мономеров $\mathrm{HF}(\mathrm{DF})$, например $-129 \mathrm{~cm}^{-1}$ в $\mathrm{H}_{2} \mathrm{O} \ldots \mathrm{HF}$ и $-71 \mathrm{~cm}^{-1}$ в $\mathrm{H}_{2} \mathrm{O}$...DF. Диагональные константы либрационных мод $v_{5}$ и валентных мод $v_{4}$ меньше констант $\chi_{1,1}$ в $2-3$ раза. При дейтерировании мономера, который участвует в колебании, значения $\chi_{i, i}$ уменьшаются приблизительно в два раза. Из недиагональных констант наибольшие значения имеют константы взаимодействия $v_{4}\left(\mathrm{~A}_{1}\right)$ и $v_{4}\left(\mathrm{~B}_{1}\right)\left(-150 \mathrm{~cm}^{-1}\right.$ в $\mathrm{H}_{2} \mathrm{O}$. . . HF и $-159 \mathrm{~cm}^{-1}$ в $\mathrm{H}_{2} \mathrm{O}$. . . DF $)$. Несколько меньшие значения имеют константы взаимодействия $v_{1}\left(\mathrm{~A}_{1}\right)$ с либрационными модами $v_{5}\left(\mathrm{~B}_{1}\right)$ и $v_{5}\left(\mathrm{~B}_{2}\right)$ (в $\mathrm{H}_{2} \mathrm{O}$...НF 105 и $87 \mathrm{~cm}^{-1}$ соответственно). При дейтерировании молекулы фтористого водорода эти ангармонические константы также уменьшаются приблизительно в два раза. Тот факт, что ангармонические значения частоты и интенсивности $v_{4}\left(\mathrm{~A}_{1}\right)$ значительно меняются при замене $\mathrm{HF}$ на DF, а спектральные параметры $v_{4}\left(\mathrm{~B}_{1}\right)$ 
практически не меняются при такой замене (табл. 2), коррелирует с аналогичными изменениями констант ангармонического взаимодействия моды $v_{1}\left(\mathrm{~A}_{1}\right)$ с модами $v_{4}\left(\mathrm{~A}_{1}\right)$ и $v_{4}\left(\mathrm{~B}_{1}\right)$. Например, в $\mathrm{D}_{2} \mathrm{O}$...НF константы взаимодействия $v_{1}\left(\mathrm{~A}_{1}\right)$ с $v_{4}\left(\mathrm{~A}_{1}\right)$ и $v_{4}\left(\mathrm{~B}_{1}\right)$ равны -4.41 и $-4.35 \mathrm{~cm}^{-1}$, а в $\mathrm{D}_{2} \mathrm{O}$...DF они равны соответственно -28.31 и $-9.87 \mathrm{~cm}^{-1}$.

\section{Вариационный расчет либрационного колебания $v_{6}\left(\mathrm{~B}_{2}\right)$ молекулы воды}

В работах $[9,10]$ колебания $v_{6}\left(\mathrm{~B}_{2}\right)$ и $v_{6}\left(\mathrm{~B}_{1}\right)$ молекулы $\mathrm{H}_{2} \mathrm{O}$ в комплексе $\mathrm{H}_{2} \mathrm{O}$...HF рассматривались как заторможенные вращательные движения большой амплитуды вокруг центров тяжести молекулы. Оператор кинетической энергии таких движений выражался через квадрат оператора углового момента и соответствующую компоненту тензора инерции. Валентные колебания $\mathrm{H}-\mathrm{F}$ и водородной связи моделировались валентными колебаниями свободной молекулы НF и гипотетической двухатомной молекулы, состоящей из центров тяжести мономеров. Взаимодействия между этими элементарными колебаниями учитывалось при помощи суперпозиции их волновых функций при решении многомерных задач. Этот подход оказался удачным, в частности, при описании колебания $v_{6}\left(\mathrm{~B}_{2}\right)$ в потенциале с двумя минимумами, когда волновая функция колебания отыскивалась в виде разложения по полиномам Лежандра. Из решения вариационного уравнения в одномерном приближении для фундаментальной частоты $v_{6}\left(\mathrm{~B}_{2}\right)$ в $\mathrm{H}_{2} \mathrm{O}$...HF было получено значение $89.4 \mathrm{~cm}^{-1}$ [10]. Решение двумерной ангармонической колебательной задачи $\left(v_{6}\left(\mathrm{~B}_{2}\right)\right.$, $\left.v_{3}\left(\mathrm{~A}_{1}\right)\right)$ привело к значению $88.4 \mathrm{~cm}^{-1}$. Незначительное изменение частоты $v_{6}\left(\mathrm{~B}_{2}\right)$ показывает, что взаимодействие $v_{6}\left(\mathrm{~B}_{2}\right)$ с валентным колебанием водородной связи слабое. Более значительным является взаимодействие $v_{6}\left(\mathrm{~B}_{2}\right)$ с валентным колебанием $\mathrm{H}-\mathrm{F}$. Решение трехмерной колебательной задачи $\left(v_{6}\left(\mathrm{~B}_{2}\right), v_{3}\left(\mathrm{~A}_{1}\right), v_{1}\left(\mathrm{~A}_{1}\right)\right)$ дает значение $v_{6}\left(\mathrm{~B}_{2}\right)=77.5 \mathrm{~cm}^{-1}[10]$, которое хорошо согласуется с экспериментальным значением $64 \pm 10 \mathrm{~cm}^{-1}$ [5]. Важным результатом работ $[9,10]$ было предсказание значения частоты горячего перехода по моде $v_{1}\left(\mathrm{~A}_{1}\right)$ с первого возбужденного состояния моды $v_{6}\left(\mathrm{~B}_{2}\right)$ более низкого, чем значение частоты фундаментального перехода. Этот факт позволил уточнить описание структуры экспериментального спектра $\mathrm{H}_{2} \mathrm{O}$. . .НF в газовой фазе. Этот аномальный факт был качественно объяснен наличием потенциального барьера для колебания $v_{6}\left(\mathrm{~B}_{2}\right)$ и зависимостью высоты этого барьера от степени возбуждения колебания $v_{1}\left(\mathrm{~A}_{1}\right)$.

Влияние колебания $v_{1}\left(\mathrm{~A}_{1}\right)$ на колебание $v_{6}\left(\mathrm{~B}_{2}\right)$ в значительной степени сводится к изменению высоты инверсионного барьера при изменении длины связи $\mathrm{H}-\mathrm{F}$. С использованием подхода, предложенного в $[9,10]$, в настоящей работе были рассчитаны потенциалы для колебания $v_{6}\left(\mathrm{~B}_{2}\right)$ в четырех рассматриваемых комплексах в предположении, что длина молекулы HF (DF) принимает равновесное значение $r_{e}$, среднее значение $\langle r\rangle_{\nu=0}$

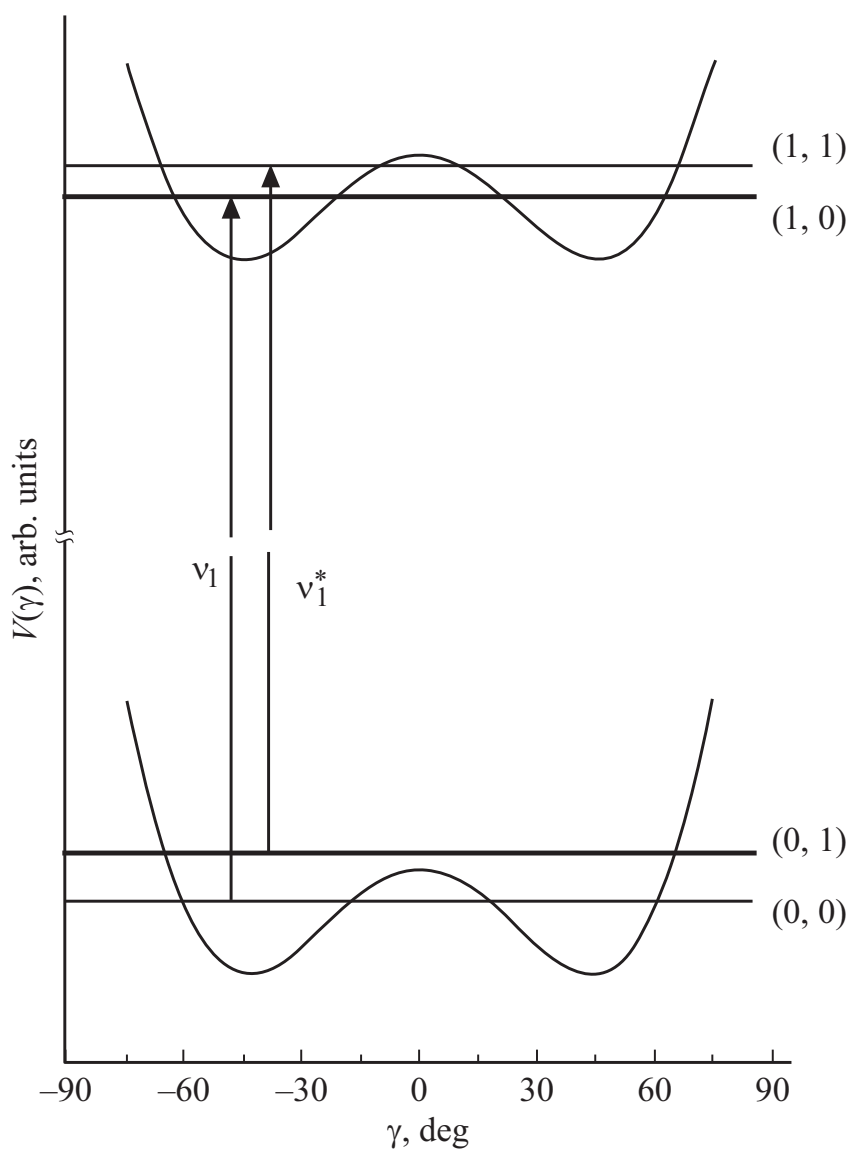

Рис. 2. Потенциальные кривые для колебания $v_{6}\left(\mathrm{~B}_{2}\right)$, вычисленные при средних значениях $r(\mathrm{HF})$ в основном и первом возбужденном состояниях валентного колебания $\mathrm{H}-\mathrm{F}$. $\gamma=\left(180^{\circ}-\theta_{1}\right) \quad($ рис. 1$),\left(v_{1}\left(\mathrm{~A}_{1}\right), v_{6}\left(\mathrm{~B}_{2}\right)\right)$ - двумерные колебательные уровни энергии, стрелки $v_{1}$ и $v_{1}^{*}$ показывают фундаментальный и горячий переходы.

по основному колебательному состоянию моды $v_{1}\left(\mathrm{~A}_{1}\right)$ и среднее значение $\langle r\rangle_{\nu=1}$ по первому возбужденному состоянию моды $v_{1}\left(\mathrm{~A}_{1}\right)$. Понятно, что $r_{e}<\langle r\rangle_{\nu=0}<\langle r\rangle_{\nu=1}$ благодаря ангармоничности колебания $v_{1}\left(\mathrm{~A}_{1}\right)$. Волновые функции и уровни энергии колебаний $v_{1}\left(\mathrm{~A}_{1}\right)$ и $v_{6}\left(\mathrm{~B}_{2}\right)$ рассчитывались вариационным методом в одномерном приближении. Таблица 3 содержит значения высоты инверсионного барьера, энергий основного $E_{0}$ и первого возбужденного $E_{1}$ уровней моды $v_{6}\left(\mathrm{~B}_{2}\right)$ и частоты $v(1 \leftarrow 0)$ перехода между колебательными уровнями $E_{0}$ и $E_{1}$, вычисленные для комплексов $\mathrm{H}_{2} \mathrm{O} \ldots \mathrm{HF}$, $\mathrm{H}_{2} \mathrm{O} \ldots \mathrm{DF}, \mathrm{D}_{2} \mathrm{O} \ldots . \mathrm{HF}$ и $\mathrm{D}_{2} \mathrm{O}$...DF. Из табл. 3 видно, что высота инверсионного барьера растет с удлинением молекулы фтористого водорода и первый возбужденный уровень $v_{6}\left(\mathrm{~B}_{2}\right)$ оказывается ниже высоты барьера. При этом резко уменьшается частота $v(1 \leftarrow 0)$. Рисунок 2 схематически изображает потенциальные кривые, вычисленные для $v_{6}\left(\mathrm{~B}_{2}\right)$ при $\langle r\rangle_{\nu=0}$ и $\langle r\rangle_{\nu=1}$ в $\mathrm{H}_{2} \mathrm{O} \ldots \mathrm{HF}$ или $\mathrm{H}_{2} \mathrm{O}$. . . DF. Вертикальные стрелки показывают фундаментальный переход $v_{1}$ по валентному колебанию фтористого водорода и горячий переход $v_{1}^{*}$. Рисунок 2 
Таблица 3. Значения высоты барьера $V_{\max }$, энергии основного $E_{0}$ и первого возбужденного $E_{1}$ состояний и частоты перехода $v$ $(1 \leftarrow 0)$ между $E_{0}$ и $E_{1}\left(\right.$ в $\left.\mathrm{cm}^{-1}\right)$, рассчитанные для колебания $v_{6}\left(\mathrm{~B}_{2}\right)$ комплексов $\mathrm{H}_{2} \mathrm{O} \ldots \mathrm{HF}, \mathrm{H}_{2} \mathrm{O} \ldots \mathrm{DF}, \mathrm{D}_{2} \mathrm{O} \ldots \mathrm{HF}$ и $\mathrm{D}_{2} \mathrm{O} \ldots \mathrm{DF}$

\begin{tabular}{|c|c|c|c|c|c|}
\hline$r(\mathrm{HF})$ или $r(\mathrm{DF})$ & Параметр & $\mathrm{H}_{2} \mathrm{O} \ldots \mathrm{HF}$ & $\mathrm{H}_{2} \mathrm{O} \ldots \mathrm{DF}$ & $\mathrm{D}_{2} \mathrm{O} \ldots \mathrm{HF}$ & $\mathrm{D}_{2} \mathrm{O} \ldots \mathrm{DF}$ \\
\hline \multirow[t]{4}{*}{$r_{e}$} & $V_{\max }$ & 123.2 & 123.2 & 127.2 & 127.2 \\
\hline & $E_{0}$ & 89.5 & 89.5 & 78.9 & 78.9 \\
\hline & $E_{1}$ & 155.9 & 155.9 & 112.7 & 112.7 \\
\hline & $v(1 \leftarrow 0)$ & 66.4 & 66.4 & 33.9 & 33.9 \\
\hline \multirow[t]{4}{*}{$\langle r\rangle_{\nu=0}$} & $V_{\max }$ & 141.2 & 136.6 & 145.4 & 137.7 \\
\hline & $E_{0}$ & 96.8 & 95.0 & 85.9 & 83.0 \\
\hline & $E_{1}$ & 157.8 & 157.3 & 115.8 & 114.6 \\
\hline & $v(1 \leftarrow 0)$ & 61.0 & 62.3 & 29.9 & 31.6 \\
\hline \multirow[t]{4}{*}{$\langle r\rangle_{\nu=1}$} & $V_{\max }$ & 183.6 & 167.0 & 188.8 & 161.0 \\
\hline & $E_{0}$ & 112.1 & 106.4 & 100.4 & 91.5 \\
\hline & $E_{1}$ & 161.3 & 160.0 & 122.4 & 118.4 \\
\hline & $v(1 \leftarrow 0)$ & 49.2 & 53.6 & 22.0 & 26.9 \\
\hline
\end{tabular}

наглядно показывает, что частота горячего перехода должна быть меньше частоты фундаментального перехода. Согласно выполненному модельному расчету, разность этих частот составляет $11.8 \mathrm{~cm}^{-1}$ в $\mathrm{H}_{2} \mathrm{O}$...НF и $8.7 \mathrm{~cm}^{-1}$ в $\mathrm{H}_{2} \mathrm{O}$...DF. Такое относительное расположение частот фундаментального и горячего переходов должно иметь место также в $\mathrm{D}_{2} \mathrm{O}$...HF и $\mathrm{D}_{2} \mathrm{O} \ldots \mathrm{DF}$. Но в этих комплексах разность частот двух переходов должна быть меньше, так как уровень $E_{1}$ при $\langle r\rangle_{\nu=0}$ тоже находится под барьером.

\section{Заключение}

В настоящей работе выполнены расчеты структуры и гармонические и ангармонические расчеты частот и интенсивностей ИК полос поглощения комплексов с водородными связями $\mathrm{H}_{2} \mathrm{O}$. . .HF, $\mathrm{H}_{2} \mathrm{O}$...DF, $\mathrm{D}_{2} \mathrm{O}$...HF и $\mathrm{D}_{2} \mathrm{O}$...DF. Был использован пакет программ Gaussian 16 [20] и метод MP2/aug-cc-pVTZ с учетом ошибки наложения базисных наборов мономеров. Ангармонические расчеты спектральных параметров выполнены с использованием колебательной теории возмущений второго порядка VPT2 [18,19]. Результаты расчетов сравниваются с немногочисленными экспериментальными данными и результатами имеющихся в литературе расчетов комплекса $\mathrm{H}_{2} \mathrm{O}$. . HF. Проанализированы тенденции в изменениях спектральных параметров при переходе от гармонического расчета к ангармоническому и при изменении изотопного состава комплексов.

Полученные в настоящей работе значения структурных параметров исследуемых комплексов находятся в хорошем согласии с наиболее точными литературными данными. Сравнение с результатами расчетов спектральных параметров мономеров, выполненных на том же уровне теории, показало, что при образовании комплексов более всего меняются частоты и интенсивности валентного колебания молекул $\mathrm{HF}$ (DF) и интенсивности валентных колебаний молекул $\mathrm{H}_{2} \mathrm{O}\left(\mathrm{D}_{2} \mathrm{O}\right)$. Было отмечено, что использование гармонических нормальных координат для расчета колебаний приводит к ошибочным результатам для либрационного колебания $v_{6}\left(\mathrm{~B}_{2}\right)$ молекулы воды как на этапе гармонического расчета, так и в ангармоническом расчете, так как нормальная координата описывает это колебание только в одном из двух минимумов потенциальной поверхности. Этот недостаток не сказывается на результатах расчета высокочастотных колебаний комплексов. Полученные в данной работе значения частот фундаментальных колебаний комплекса $\mathrm{H}_{2} \mathrm{O}$...HF находятся в хорошем согласии с результатами расчета [16] с более полным набором атомных функций aug-cc-pV5Z. Значения частот валентного колебания $v_{1}\left(\mathrm{~A}_{1}\right)$ и либрационных колебаний $v_{5}\left(\mathrm{~B}_{1}\right)$ и $v_{5}\left(\mathrm{~B}_{2}\right)$, рассчитанные в настоящей работе в ангармоническом приближении, несколько выше соответствующих результатов матричного эксперимента [8], что объяснимо влиянием матричного окружения. С использованием полного потенциала с двумя ямами для либрационного колебания $v_{6}\left(\mathrm{~B}_{2}\right)$ были выполнены одномерные вариационные расчеты фундаментальной частоты этого колебания для всех рассмотренных комплексов, находящихся в основном и первом возбужденном состояниях валентного колебания $v_{1}\left(\mathrm{~A}_{1}\right)$. Построенный на основе этих данных рисунок наглядно объясняет причину более высокого значения частоты фундаментального перехода по моде $v_{1}\left(\mathrm{~A}_{1}\right)$ по сравнению с частотой горячего перехода по этой моде с первого возбужденного состояния моды $v_{6}\left(\mathrm{~B}_{2}\right)$.

\section{Финансирование работы}

Работа частично выполнена при финансовой поддержке Российского фонда фундаментальных исследований, грант № 18-03-00520.

\section{Конфликт интересов}

Авторы заявляют, что у них нет конфликта интересов. 


\section{Список литературы}

[1] Preller M., Grunenberg J., Bulychev V.P., Bulanin M.O. // J. Chem. Phys. 2011. V. 134. P. 174302.

[2] Bulychev V.P., Svishcheva E.A., Tokhadze K.G. // Spectrochim. Acta A. 2014. V. 117. P. 679.

[3] Bulychev V.P., Buturlimova M.V., Tokhadze K.G. // J. Chem. Phys. 2018. V. 149. P. 104306.

[4] Bevan J.W., Kisiel Z., Legon A.C., Millen D.J, Rogers S.C. // Proc. Roy. Soc. London A. 1980. V. 372. P. 441-451.

[5] Kisiel Z., Legon A.C., Millen D.J. // Proc. Roy. Soc. London A.1982. V. 381. P. 419-442.

[6] Thomas R.K. // Proc. Roy. Soc. London A. 1971. V. 325. P. $133-149$.

[7] Thomas R.K. // Proc. Roy. Soc. London A. 1975. V. 344. P. 579-592.

[8] Andrews L., Johnson G.L. // J. Chem. Phys. 1983. V. 79. P. 3670.

[9] Бульчев В.П., Громова Е.И., Тохадзе К.Г. // Опт. и спектр. 2004. T. 96. C. 774.

[10] Bulychev V.P., Grigoriev I.M., Gromova E.I., Tokhadze K.G. // Phys. Chem. Chem. Phys. 2005. V. 7. P. 2266.

[11] Goubet M., Madebene B., Lewerenz M. // Chimia. 2004. V. 58. P. 291.

[12] Szczesniak M.M., Scheiner S., Bouteiller Y. // J. Chem. Phys. 1984. V. 81. P. 5024.

[13] Latajka Z., Scheiner S. // J. Comp. Chem. 1987. V. 8. P. 674.

[14] Novoa J.J., Planas M., Whangbo M.-H., Williams J.M. // Chem. Phys. 1994. V. 186. P. 175.

[15] Demaison J., Lievin J. // Mol. Phys. 2008. V. 106. P. 1249.

[16] Sexton Th.M., Howard J.C., Tschumper G.S. // J. Phys. Chem. A. 2018. V. 122. P. 4902.

[17] Loreau J., Kalugina Yu.N., Faure A., van der Avoird A., Lique F. // J. Chem. Phys. 2020. V. 153. P. 214301.

[18] Barone V. // J. Chem. Phys. 2005. V. 122. P. 014108.

[19] Bloino J. // J. Phys. Chem. A. 2015. V. 119. P. 5269.

[20] Gaussian 16, Revision A.03. Frisch M.J., Trucks G.W., Schlegel H.B., Scuseria G.E., Robb M.A., Cheeseman J.R., Scalmani G., Barone V., Petersson G.A., Nakatsuji H., Li X., Caricato M., Marenich V., Bloino J., Janesko B.G., Gomperts R., Mennucci B., Hratchian H.P., Ortiz J.V., Izmaylov A.F., Sonnenberg J.L., Williams-Young D., Ding F., Lipparini F., Egidi F., Goings J., Peng B., Petrone A., Henderson T., Ranasinghe D., Zakrzewski V.G., Gao J., Rega N., Zheng G., Liang W., Hada M., Ehara M., Toyota K., Fukuda R., Hasegawa J., Ishida M., Nakajima T., Honda Y., Kitao O., Nakai H., Vreven T., Throssell K., Montgomery J.A., Jr., Peralta J.E., Ogliaro F., Bearpark M.J., Heyd J.J., Brothers E.N., Kudin K.N., Staroverov V.N., Keith T.A., Kobayashi R., Normand J., Raghavachari K., Rendell A.P., Burant J.C., Iyengar S.S., Tomasi J., Cossi M., Millam J.M., Klene M., Adamo C., Cammi R., Ochterski J.W., Martin R.L., Morokuma K., Farkas O., Foresman J.B., Fox D.J. Gaussian, Inc., Wallingford CT, 2016.

[21] Булычев В.П., Енгалычева Е.А., Тохадзе К.Г. // Опт. и спектр. 2019. Т. 126. С. 404.

[22] Oswald S., Suhm M.A. // Phys. Chem. Chem. Phys. 2019. V. 21. P. 18799. 